\title{
THE HIGHER ORDER REGULARITY DIRICHLET PROBLEM FOR ELLIPTIC SYSTEMS IN THE UPPER-HALF SPACE
}

\author{
JOSÉ MARÍA MARTELL, DORINA MITREA, IRINA MITREA, AND MARIUS MITREA
}

\begin{abstract}
We identify a large class of constant (complex) coefficient, second order elliptic systems for which the Dirichlet problem in the upper-half space with data in $L^{p}$-based Sobolev spaces, $1<p<\infty$, of arbitrary smoothness $\ell$, is well-posed in the class of functions whose nontangential maximal operator of their derivatives up to, and including, order $\ell$ is $L^{p}$-integrable. This class includes all scalar, complex coefficient elliptic operators of second order, as well as the Lamé system of elasticity, among others.
\end{abstract}

\section{INTRODUCTION}

Let $M$ be a fixed positive integer and consider the second-order, $M \times M$ system, with constant complex coefficients, written as

$$
L u:=\left(\partial_{r}\left(a_{r s}^{\alpha \beta} \partial_{s} u_{\beta}\right)\right)_{1 \leq \alpha \leq M}
$$

when acting on a $\mathscr{C}^{2}$ vector valued function $u=\left(u_{\beta}\right)_{1 \leq \beta \leq M}$. A standing assumption for this paper is that $L$ is elliptic, in the sense that there exists a real number $\kappa_{o}>0$ such that the following Legendre-Hadamard condition is satisfied (here and elsewhere, the usual convention of summation over repeated indices is used)

$$
\begin{gathered}
\operatorname{Re}\left[a_{r s}^{\alpha \beta} \xi_{r} \xi_{s} \overline{\eta_{\alpha}} \eta_{\beta}\right] \geq \kappa_{o}|\xi|^{2}|\eta|^{2} \text { for every } \\
\xi=\left(\xi_{r}\right)_{1 \leq r \leq n} \in \mathbb{R}^{n} \text { and } \eta=\left(\eta_{\alpha}\right)_{1 \leq \alpha \leq M} \in \mathbb{C}^{M} .
\end{gathered}
$$

The $L^{p}$-Dirichlet boundary problem associated with the operator $L$ in the upperhalf space is formulated as $L u=0$ in $\mathbb{R}_{+}^{n},\left.u\right|_{\partial \mathbb{R}_{+}^{n}} ^{n . t .}=f \in L^{p}\left(\mathbb{R}^{n-1}\right)$, and $\mathcal{N} u \in$ $L^{p}\left(\partial \mathbb{R}_{+}^{n}\right)$. Here and elsewhere, $\mathcal{N}$ denotes the nontangential maximal operator, while $\left.u\right|_{\partial \mathbb{R}_{+}^{n}} ^{\text {n.t. }}$ stands for the non-tangential trace of $u$ onto $\partial \mathbb{R}_{+}^{n}$ (for precise definitions see (2.2) and (2.5)). While in the particular case $L=\Delta$, the Laplacian in $\mathbb{R}^{n}$, this

Date: October 30, 2012. Revised: March 22, 2013.

2010 Mathematics Subject Classification. Primary: 35B65, 35J45, 35J57. Secondary: 35C15, 74B05, 74G05.

Key words and phrases. Higher order Dirichlet problem, nontangential maximal function, second order elliptic system, Poisson kernel, Lamé system.

The first author has been supported in part by MINECO Grant MTM2010-16518 and ICMAT Severo Ochoa project SEV-2011-0087, the second author has been supported in part by a Simons Foundation grant 200750 and by a University of Missouri research leave, the third author has been supported in part by US NSF grant 0547944 . The fourth author has been supported in part by the Simons Foundation grant 281566. This work has been possible thanks to the support and hospitality of Temple University (USA), ICMAT, Consejo Superior de Investigaciones Cientificas (Spain) and the Universidad Autónoma de Madrid (Spain). The authors express their gratitude to these institutions. 
boundary value problem has been treated at length in many monographs, including [3], [16], [17], to give just a few examples, much remains to be done.

Here we are interested in identifying a class of elliptic systems $L$ for which the Dirichlet problem in the upper-half space is well-posed for boundary data belonging to higher-order smoothness spaces, such as $L_{\ell}^{p}\left(\mathbb{R}^{n-1}\right)$, the $L^{p}$-based Sobolev space in $\mathbb{R}^{n-1}$ of order $\ell \in \mathbb{N}_{0}$, with $p \in(1, \infty)$. In such a scenario, we shall demand that one retains nontangential control of higher-order derivatives of the solution. More precisely, given any $\ell \in \mathbb{N}_{0}$, we formulate the $\ell$-th order Dirichlet boundary value problem for $L$ in $\mathbb{R}_{+}^{n}$ as follows

$$
\left\{\begin{array}{l}
L u=0 \text { in } \mathbb{R}_{+}^{n} \text { and }\left.u\right|_{\partial \mathbb{R}_{+}^{n}} ^{n . t .}=f \in L_{\ell}^{p}\left(\mathbb{R}^{n-1}\right), \\
\mathcal{N}\left(\nabla^{k} u\right) \in L^{p}\left(\partial \mathbb{R}_{+}^{n}\right) \text { for } k \in\{0,1, \ldots, \ell\},
\end{array}\right.
$$

where $\nabla^{k} u$ denotes the vector with components $\left(\partial^{\alpha} u\right)_{|\alpha|=k}$. No concrete case of (1.3) has been dealt with for arbitrary values of the smoothness parameter $\ell$, so considering even $L=\Delta$ in such a setting is new. In fact, we are able to treat differential operators that are much more general than the Laplacian, again, in the context when the boundary data exhibit an arbitrary amount of regularity, measured on the $L^{p}$-based Sobolev scale.

In dealing with (1.3), the starting point is the fact that, as known from the seminal work of S. Agmon, A. Douglis, and L. Nirenberg in [1] and [2], every constant coefficient elliptic operator $L$ has a Poisson kernel $P^{L}$, an object whose properties mirror the most basic characteristics of the classical harmonic Poisson kernel

$$
P^{\Delta}\left(x^{\prime}\right):=\frac{2}{\omega_{n-1}} \frac{1}{\left(1+\left|x^{\prime}\right|^{2}\right)^{\frac{n}{2}}} \quad \forall x^{\prime} \in \mathbb{R}^{n-1},
$$

where $\omega_{n-1}$ is the area of the unit sphere $S^{n-1}$ in $\mathbb{R}^{n}$. In particular, using the notation $F_{t}\left(x^{\prime}\right):=t^{1-n} F\left(x^{\prime} / t\right)$ for each $t>0$ where $F$ is a generic function defined in $\mathbb{R}^{n-1}$, we have

$$
\left|P_{t}^{L}\left(x^{\prime}\right)\right| \leq C \frac{t}{\left(t^{2}+\left|x^{\prime}\right|^{2}\right)^{\frac{n}{2}}} \quad \forall x^{\prime} \in \mathbb{R}^{n-1}, \quad \forall t>0 .
$$

Then, given any $f \in L^{p}\left(\mathbb{R}^{n-1}\right), 1<p<\infty$, if $\mathcal{M}$ stands for the Hardy-Littlewood maximal operator in $\mathbb{R}^{n-1}$, the function

$$
u\left(x^{\prime}, t\right):=\left(P_{t}^{L} * f\right)\left(x^{\prime}\right), \quad \forall\left(x^{\prime}, t\right) \in \mathbb{R}_{+}^{n},
$$

satisfies $L u=0$ in $\mathbb{R}_{+}^{n}$ as well as $\left.u\right|_{\partial \mathbb{R}_{+}^{n}} ^{\text {n.t. }}=f$ a.e. in $\mathbb{R}^{n-1}$, and

$$
(\mathcal{N} u)\left(x^{\prime}\right) \leq C(\mathcal{M} f)\left(x^{\prime}\right), \quad \forall x^{\prime} \in \mathbb{R}^{n-1} .
$$

In turn, the pointwise estimate (1.7) and the boundedness of $\mathcal{M}$ on $L^{p}\left(\mathbb{R}^{n-1}\right)$, $1<p<\infty$, can be used to show, much as in the case for the Laplacian, that $u$ from (1.6) solves the $L^{p}$-Dirichlet problem in the upper half-space for any given constant coefficient elliptic operator $L$. This corresponds to the case $\ell=0$ in (1.3).

This being said, it is unclear whether the Agmon-Douglis-Nirenberg Poisson kernel for a generic elliptic operator $L$ continues to work just as well in the setting when the boundary data is assumed to have higher order regularity. The issue is that, in this scenario, one is required to estimate the size of the nontangential maximal operator of iterated gradients of the solution. For such a goal, in order to make use of the higher order regularity assumption on the boundary data, one necessarily 
must find a way of passing generic derivatives inside the convolution (1.6), while at the same time allowing kernels, of an auxiliary nature, to take the role of the original Poisson kernel. The caveat is that the nontangential maximal function of convolutions with these auxiliary kernels should have appropriate control, a matter which may not always be ensured.

To better understand the nature of this difficulty, consider the case of (1.3) with $\ell=1$, a scenario in which one still looks for a solution as in (1.6) (keeping in mind that now $f$ belongs to the Sobolev space $\left.L_{1}^{p}\left(\mathbb{R}^{n-1}\right), 1<p<\infty\right)$. As far as estimating $\mathcal{N}\left(\partial_{x_{j}} u\right)$ is concerned, it is clear from (1.6) that only the derivative in the normal direction (i.e., for $\partial_{t} \equiv \partial_{x_{n}}$ ) is potentially problematic. In the absence of additional information about the nature of the Poisson kernel $P^{L}$ one tool that naturally presents itself is a general identity, valid for any function $F \in \mathscr{C}^{1}\left(\mathbb{R}^{n-1}\right)$, to the effect that

$$
\partial_{t}\left[F_{t}\left(x^{\prime}\right)\right]=-\sum_{j=1}^{n-1} \partial_{x_{j}}\left[\left(x_{j} F\left(x^{\prime}\right)\right)_{t}\right] \text { for every }\left(x^{\prime}, t\right) \in \mathbb{R}_{+}^{n} .
$$

For $u$ as in (1.6), this permits us to express

$$
\begin{aligned}
\partial_{t}\left[u\left(x^{\prime}, t\right)\right] & =\partial_{t}\left[\left(P_{t}^{L} * f\right)\left(x^{\prime}\right)\right]=-\sum_{j=1}^{n-1} \partial_{x_{j}}\left[\left(R_{t}^{(j)} * f\right)\left(x^{\prime}\right)\right] \\
& =-\sum_{j=1}^{n-1}\left[R_{t}^{(j)} *\left(\partial_{j} f\right)\right]\left(x^{\prime}\right) \text { for every }\left(x^{\prime}, t\right) \in \mathbb{R}_{+}^{n},
\end{aligned}
$$

where the auxiliary kernels $R^{(j)}, 1 \leq j \leq n-1$, are given by

$$
R^{(j)}\left(x^{\prime}\right):=x_{j} P^{L}\left(x^{\prime}\right), \text { for every } x^{\prime} \in \mathbb{R}^{n-1} .
$$

Superficially, the terms in the right-most side of (1.9) appear to have the same type of structure as the original function $u$ in (1.6) (since $\partial_{j} f \in L^{p}\left(\mathbb{R}^{n-1}\right)$ ), which raises the prospect of handling them as in (1.7). However, such optimism is not justified since the auxiliary kernels $R^{(j)}$ have a fundamentally different behavior at infinity than the original $P^{L}$. Concretely, in place of (1.5) we now have

$$
\left|R_{t}^{(j)}\left(x^{\prime}\right)\right| \leq C \frac{\left|x_{j}\right|}{\left(t^{2}+\left|x^{\prime}\right|^{2}\right)^{\frac{n}{2}}}, \quad \forall x^{\prime} \in \mathbb{R}^{n-1}, \quad \forall t>0 .
$$

In particular, $R_{t}^{(j)}\left(x^{\prime}\right)$ only decays as $\left|x^{\prime}\right|^{1-n}$ at infinity, for each $t>0$ fixed, so the analogue of (1.7) in this case, i.e., the pointwise estimate

$$
\mathcal{N}\left(\partial_{t} u\right) \leq C \mathcal{M}\left(\nabla^{\prime} f\right) \text { in } \mathbb{R}^{n-1},
$$

where $\nabla^{\prime}$ denotes the gradient in $\mathbb{R}^{n-1}$, is rendered hopeless. This being said, the usual technology used in the proof of Cotlar's inequality may be employed to show that in place of (1.12) one nonetheless has

$$
\mathcal{N}\left(\partial_{t} u\right) \leq C \sum_{j=1}^{n-1} T_{\star}^{(j)}\left(\partial_{j} f\right)+C \mathcal{M}\left(\nabla^{\prime} f\right) \quad \text { in } \quad \mathbb{R}^{n-1},
$$


where $T_{\star}^{(j)}$ is the maximal singular integral operator acting on a generic function $g$ defined in $\mathbb{R}^{n-1}$ according to

$$
T_{\star}^{(j)} g\left(x^{\prime}\right):=\sup _{\varepsilon>0}\left|\int_{\left|x^{\prime}-y^{\prime}\right|>\varepsilon} k_{j}\left(x^{\prime}-y^{\prime}\right) g\left(y^{\prime}\right) d y^{\prime}\right|, \quad x^{\prime} \in \mathbb{R}^{n-1},
$$

where the kernel $k_{j}$ is given by

$$
k_{j}\left(x^{\prime}\right):=\left.x_{j} \partial_{t}\left[P_{t}^{L}\left(x^{\prime}\right)\right]\right|_{t=0}, \quad x^{\prime} \in \mathbb{R}^{n-1} \backslash\left\{0^{\prime}\right\} .
$$

In concert with the fact that each $k_{j}$ has the right amount of regularity and homogeneity, i.e.,

$$
\begin{aligned}
& k_{j} \in \mathscr{C}^{\infty}\left(\mathbb{R}^{n-1} \backslash\left\{0^{\prime}\right\}\right), \quad k_{j}\left(\lambda x^{\prime}\right)=\lambda^{1-n} k_{j}\left(x^{\prime}\right) \\
& \text { for every } \lambda>0 \text { and every } x^{\prime} \in \mathbb{R}^{n-1} \backslash\left\{0^{\prime}\right\},
\end{aligned}
$$

estimate (1.13) then steers the proof of bounding the $L^{p}$ norm of $\mathcal{N}\left(\partial_{t} u\right)$ in the direction of Calderón-Zygmund theory. However, what is needed for the latter to apply is a suitable cancellation condition for the kernels $k_{j}$, say

$$
\int_{S^{n-2}} k_{j}\left(\omega^{\prime}\right) d \omega^{\prime}=0, \quad \forall j \in\{1, \ldots, n-1\} .
$$

Under the mere ellipticity assumption on $L$ there is no reason to expect that a cancellation condition such as (1.17) happens, so extra assumptions, of an algebraic nature, need to be imposed to ensure its validity. In the sequel, we identify a class of operators (cf. Definition 3.7) for which the respective kernels $k_{j}$ are odd, thus (1.17) holds. A natural issue to consider is whether condition (1.17) would, on its own, ensure well-posedness for (1.3). The answer is no, as it may be seen by looking at the case of (1.3) with $\ell=2$. This time, the boundary datum $f$ is assumed to belong to $L_{2}^{p}\left(\mathbb{R}^{n-1}\right)$ and one is required to estimate the $L^{p}$ norm of $\mathcal{N}\left(\partial_{t}^{2} u\right)$. By running the above procedure, one now obtains (based on (1.8) and (1.9))

$$
\begin{aligned}
\partial_{t}^{2}\left[u\left(x^{\prime}, t\right)\right] & =-\sum_{j=1}^{n-1} \partial_{t}\left[R_{t}^{(j)} *\left(\partial_{j} f\right)\right]\left(x^{\prime}\right) \\
& =\sum_{i=1}^{n-1} \sum_{j=1}^{n-1}\left[R_{t}^{(i j)} *\left(\partial_{i} \partial_{j} f\right)\right]\left(x^{\prime}\right) \text { for every }\left(x^{\prime}, t\right) \in \mathbb{R}_{+}^{n},
\end{aligned}
$$

where the second generation auxiliary kernels $R^{(i j)}, 1 \leq i, j \leq n-1$, are given by

$$
R^{(i j)}\left(x^{\prime}\right):=x_{i} x_{j} P^{L}\left(x^{\prime}\right), \text { for every } x^{\prime} \in \mathbb{R}^{n-1} .
$$

However, these kernels exhibit a worse decay condition at infinity than their predecessors in (1.11), since now we only have

$$
\left|R_{t}^{(i j)}\left(x^{\prime}\right)\right| \leq C \frac{\left|x_{i} x_{j}\right|}{\left(t^{2}+\left|x^{\prime}\right|^{2}\right)^{\frac{n}{2}}}, \quad \forall x^{\prime} \in \mathbb{R}^{n-1}, \quad \forall t>0 .
$$

This rules out, from the outset, the possibility of involving the Calderón-Zygmund theory in the proceedings, thus rendering condition (1.17) irrelevant for the case $\ell=2$. Of course, in the context of larger values of $\ell$ one is faced with similar issues.

In summary, an approach based solely on generic qualitative properties of elliptic second order operators runs into insurmountable difficulties, and the above analysis makes the case for the necessity of additional algebraic assumptions on the nature of 
the operator $L$, without which the well-posedness of (1.3) is not generally expected for all $\ell \in \mathbb{N}_{0}$.

In this paper, we identify a large class of second order elliptic operators for which a version of the procedure outlined above may be successfully implemented. Using a piece of terminology formulated precisely in the body of the paper, these are the operators $L$ possessing a distinguished coefficient tensor (see Definition 3.7). Under such a condition, the auxiliary kernels referred to earlier become manageable and this eventually leads to the well-posedness of the higher order regularity Dirichlet problem as formulated in (1.3). See Theorem 4.1 which is the main result of the paper. In the last section, we illustrate the scope of the techniques developed here by proving that such an approach works for any constant (complex) coefficient scalar elliptic operator, as well as for the Lamé system of elasticity. In fact, even in the case of the Laplacian, our well-posedness result for the higher order Dirichlet problem in the upper-half space is new. In closing, we also point out that the same circle of ideas works equally well for other partial differential equations of basic importance in mathematical physics, such as the Stokes system of hydrodynamics, the Maxwell system of electromagnetics, and the Dirac operator of quantum theory (more on this may be found in the forthcoming monograph [8]).

\section{Preliminaries}

Throughout, we let $\mathbb{N}$ stand for the collection of all strictly positive integers, and set $\mathbb{N}_{0}:=\mathbb{N} \cup\{0\}$. Also, fix $n \in \mathbb{N}$ with $n \geq 2$. We shall work in the upper-half space

$$
\mathbb{R}_{+}^{n}:=\left\{x=\left(x^{\prime}, x_{n}\right) \in \mathbb{R}^{n}=\mathbb{R}^{n-1} \times \mathbb{R}: x_{n}>0\right\},
$$

whose topological boundary $\partial \mathbb{R}_{+}^{n}=\mathbb{R}^{n-1} \times\{0\}$ will be frequently identified with the horizontal hyperplane $\mathbb{R}^{n-1}$ via $\left(x^{\prime}, 0\right) \equiv x^{\prime}$. Fix a number $\kappa>0$ and for each boundary point $x^{\prime} \in \partial \mathbb{R}_{+}^{n}$ introduce the conical nontangential approach region

$$
\Gamma\left(x^{\prime}\right):=\Gamma_{\kappa}\left(x^{\prime}\right):=\left\{y=\left(y^{\prime}, t\right) \in \mathbb{R}_{+}^{n}:\left|x^{\prime}-y^{\prime}\right|<\kappa t\right\} .
$$

Given a vector-valued function $u: \mathbb{R}_{+}^{n} \rightarrow \mathbb{C}^{M}$, define the nontangential maximal function of $u$ by

$$
(\mathcal{N} u)\left(x^{\prime}\right):=\left(\mathcal{N}_{\kappa} u\right)\left(x^{\prime}\right):=\sup \left\{|u(y)|: y \in \Gamma_{\kappa}\left(x^{\prime}\right)\right\}, \quad x^{\prime} \in \partial \mathbb{R}_{+}^{n} .
$$

As is well-known, for every $\kappa, \kappa^{\prime}>0$ and $p \in(0, \infty)$ there exist finite constants $C_{0}, C_{1}>0$ such that

$$
C_{0}\left\|\mathcal{N}_{\kappa} u\right\|_{L^{p}\left(\partial \mathbb{R}_{+}^{n}\right)} \leq\left\|\mathcal{N}_{\kappa^{\prime}} u\right\|_{L^{p}\left(\partial \mathbb{R}_{+}^{n}\right)} \leq C_{1}\left\|\mathcal{N}_{\kappa} u\right\|_{L^{p}\left(\partial \mathbb{R}_{+}^{n}\right)},
$$

for each function $u$. Whenever meaningful, we also define

$$
\left.u\right|_{\partial \mathbb{R}_{+}^{n}} ^{n . t .}\left(x^{\prime}\right):=\lim _{\Gamma_{\kappa}\left(x^{\prime}\right) \ni y \rightarrow\left(x^{\prime}, 0\right)} u(y) \text { for } x^{\prime} \in \partial \mathbb{R}_{+}^{n} .
$$

For each $p \in(1, \infty)$ and $k \in \mathbb{N}_{0}$ denote by $L_{k}^{p}\left(\mathbb{R}^{n-1}\right)$ the classical Sobolev space of order $k$ in $\mathbb{R}^{n-1}$, consisting of functions from $L^{p}\left(\mathbb{R}^{n-1}\right)$ whose distributional derivatives up to order $k$ are in $L^{p}\left(\mathbb{R}^{n-1}\right)$. This becomes a Banach space when equipped with the natural norm

$$
\|f\|_{L_{k}^{p}\left(\mathbb{R}^{n-1}\right)}:=\|f\|_{L^{p}\left(\mathbb{R}^{n-1}\right)}+\sum_{|\alpha| \leq k}\left\|\partial^{\alpha} f\right\|_{L^{p}\left(\mathbb{R}^{n-1}\right)}, \quad \forall f \in L_{k}^{p}\left(\mathbb{R}^{n-1}\right) .
$$


Let $L$ be an elliptic operator as in (1.1)-(1.2). Call $A:=\left(a_{r s}^{\alpha \beta}\right)_{\alpha, \beta, r, s}$ the coefficient tensor of $L$. To emphasize the dependence of $L$ on $A$, let us agree to write $L_{A}$ in place of $L$ whenever necessary. In general, there are multiple ways of expressing a given system $L$ as in (1.1). Indeed, if for any given $A=\left(a_{r s}^{\alpha \beta}\right)_{\alpha, \beta, r, s}$, we define $A_{\mathrm{sym}}:=\left(\frac{1}{2}\left(a_{r s}^{\alpha \beta}+a_{s r}^{\alpha \beta}\right)\right)_{\alpha, \beta, r, s}$, then

$$
L_{A_{1}}=L_{A_{2}} \Longleftrightarrow\left(A_{1}-A_{2}\right)_{\mathrm{sym}}=0 .
$$

These considerations suggest introducing

$$
\mathfrak{A}_{L}:=\left\{A=\left(a_{r s}^{\alpha \beta}\right)_{\substack{1 \leq r, s \leq n \\ 1 \leq \alpha, \beta \leq M}} \in \mathbb{C}^{n M} \times \mathbb{C}^{n M}: L=L_{A}\right\} .
$$

It follows from (2.7) that if the original coefficient tensor of $L$ satisfies the LegendreHadamard ellipticity condition (1.2) then any other coefficient tensor in $\mathfrak{A}_{L}$ does so. In other words, the Legendre-Hadamard ellipticity condition is an intrinsic property of the differential operator being considered, which does not depend on the choice of a coefficient tensor used to represent this operator.

Given a system $L$ as in (1.1), let $L^{\top}$ be the transposed of $L$, i.e., the $M \times M$ system of differential operators satisfying

$$
\int_{\mathbb{R}^{n}}\langle L u, v\rangle d \mathscr{L}^{n}=\int_{\mathbb{R}^{n}}\left\langle u, L^{\top} v\right\rangle d \mathscr{L}^{n}, \quad \forall u, v \in \mathscr{C}_{c}^{\infty}\left(\mathbb{R}^{n}\right), \mathbb{C}^{M} \text {-valued, }
$$

where $\mathscr{L}^{n}$ stands for the Lebesgue measure in $\mathbb{R}^{n}$. A moment's reflection then shows that, if $L$ is as in (1.1), then

$$
L^{\top} u=\left(\partial_{r}\left(a_{s r}^{\beta \alpha} \partial_{s} u_{\beta}\right)\right)_{1 \leq \alpha \leq M}, \quad \forall u=\left(u_{\beta}\right)_{1 \leq \beta \leq M} \in \mathscr{C}^{2}\left(\mathbb{R}^{n}\right) .
$$

That is, if $A^{\top}:=\left(a_{s r}^{\beta \alpha}\right)_{\substack{1 \leq r, s \leq n \\ 1 \leq \alpha, \beta \leq M}}$ denotes the transpose of $A=\left(a_{r s}^{\alpha \beta}\right)_{\substack{1 \leq r, s \leq n \\ 1 \leq \alpha, \beta \leq M}}$, formula (2.10) amounts to saying that $\left(L_{A}\right)^{\top}=L_{A^{\top}}$.

The theorem below summarizes properties of a distinguished fundamental solution of the operator $L$. It builds on the work carried out in various degrees of generality in [5, pp. 72-76], [4, p. 169], [12], [11, p. 104], and a proof in the present formulation may be found in [9], [10].

Theorem 2.1. Assume that $L$ is an $M \times M$ elliptic, second order system in $\mathbb{R}^{n}$, with complex constant coefficients as in (1.1). Then there exists a matrix $E=$ $\left(E_{\alpha \beta}\right)_{1 \leq \alpha, \beta \leq M}$ whose entries are tempered distribution in $\mathbb{R}^{n}$ and such that the following properties hold:

(a) For each $\alpha, \beta \in\{1, \ldots, M\}, E_{\alpha \beta} \in \mathscr{C}^{\infty}\left(\mathbb{R}^{n} \backslash\{0\}\right)$ and $E_{\alpha \beta}(-x)=E_{\alpha \beta}(x)$ for all $x \in \mathbb{R}^{n} \backslash\{0\}$.

(b) If $\delta_{y}$ stands for Dirac's delta distribution with mass at $y$ then for each indices $\alpha, \beta \in\{1, \ldots, M\}$, and every $x, y \in \mathbb{R}^{n}$,

$$
\partial_{x_{r}} a_{r s}^{\alpha \gamma} \partial_{x_{s}}\left[E_{\gamma \beta}(x-y)\right]= \begin{cases}0 & \text { if } \alpha \neq \beta \\ \delta_{y}(x) & \text { if } \alpha=\beta .\end{cases}
$$

(c) For each $\alpha, \beta \in\{1, \ldots, M\}$, one has

$$
E_{\alpha \beta}(x)=\Phi_{\alpha \beta}(x)+c_{\alpha \beta} \ln |x|, \quad \forall x \in \mathbb{R}^{n} \backslash\{0\},
$$


where $\Phi_{\alpha \beta} \in \mathscr{C}^{\infty}\left(\mathbb{R}^{n} \backslash\{0\}\right)$ is a homogeneous function of degree $2-n$, and the matrix $\left(c_{\alpha \beta}\right)_{1 \leq \alpha, \beta \leq M} \in \mathbb{C}^{M \times M}$ is identically zero when $n \geq 3$.

(d) For each $\gamma \in \mathbb{N}_{0}^{n}$ there exists a finite constant $C_{\gamma}>0$ such that for each $x \in \mathbb{R}^{n} \backslash\{0\}$

$$
\left|\partial^{\gamma} E(x)\right| \leq\left\{\begin{array}{l}
\frac{C_{\gamma}}{|x|^{n+|\gamma|-2}} \quad \text { if either } n \geq 3, \text { or } n=2 \text { and }|\gamma|>0, \\
C_{0}(1+|\ln | x||) \quad \text { if } n=2 \text { and }|\gamma|=0 .
\end{array}\right.
$$

(e) When restricted to $\mathbb{R}^{n} \backslash\{0\}$, the (matrix-valued) distribution $\widehat{E}$ is a $\mathscr{C}^{\infty}$ function and, with "hat" denoting the Fourier transform in $\mathbb{R}^{n}$,

$$
\widehat{E}(\xi)=-\left[\left(\xi_{r} \xi_{s} a_{r s}^{\alpha \beta}\right)_{1 \leq \alpha, \beta \leq M}\right]^{-1} \text { for each } \xi \in \mathbb{R}^{n} \backslash\{0\} .
$$

$(f)$ One can assign to each elliptic differential operator $L$ as in (1.1) a fundamental solution $E^{L}$ which satisfies (a)-(e) above and, in addition, $\left(E^{L}\right)^{\top}=E^{L^{\top}}$, where the superscript $\top$ denotes transposition.

(g) In the particular case $M=1$, i.e., in the situation when $L=\operatorname{div} A \nabla$ for some matrix $A=\left(a_{r s}\right)_{1 \leq r, s \leq n} \in \mathbb{C}^{n \times n}$, an explicit formula for the fundamental solution $E$ of $L$ is

$$
E(x)= \begin{cases}-\frac{1}{(n-2) \omega_{n-1} \sqrt{\operatorname{det}\left(A_{\mathrm{sym}}\right)}}\left\langle\left(A_{\mathrm{sym}}\right)^{-1} x, x\right\rangle^{\frac{2-n}{2}} & \text { if } n \geq 3, \\ \frac{1}{4 \pi \sqrt{\operatorname{det}\left(A_{\mathrm{sym}}\right)} \log \left(\left\langle\left(A_{\mathrm{sym}}\right)^{-1} x, x\right\rangle\right)} & \text { if } n=2,\end{cases}
$$

for $x \in \mathbb{R}^{n} \backslash\{0\}$. Here, log denotes the principal branch of the complex logarithm function (defined by the requirement that $z^{t}=e^{t \log z}$ holds for every $z \in \mathbb{C} \backslash(-\infty, 0]$ and every $t \in \mathbb{R})$.

\section{POISSON KERNELS}

In this section we discuss the notion of Poisson kernel in $\mathbb{R}_{+}^{n}$ for an operator $L$ as in (1.1)-(1.2). We also identify a subclass of these Poisson kernels, which we call special Poisson kernels, that plays a significant role in the treatment of boundary value problems.

Definition 3.1 (Poisson kernel for $L$ in $\mathbb{R}_{+}^{n}$ ). Let $L$ be a second order elliptic system with complex coefficients as in (1.1)-(1.2). A Poisson kernel for $L$ in $\mathbb{R}_{+}^{n}$ is a matrix-valued function $P=\left(P_{\alpha \beta}\right)_{1 \leq \alpha, \beta \leq M}: \mathbb{R}^{n-1} \rightarrow \mathbb{C}^{M \times M}$ such that:

(a) there exists $C \in(0, \infty)$ such that $\left|P\left(x^{\prime}\right)\right| \leq \frac{C}{\left(1+\left|x^{\prime}\right|^{2}\right)^{\frac{n}{2}}}$ for each $x^{\prime} \in \mathbb{R}^{n-1}$;

(b) one has $\int_{\mathbb{R}^{n-1}} P\left(x^{\prime}\right) d x^{\prime}=I_{M \times M}$, the $M \times M$ identity matrix;

(c) if $K\left(x^{\prime}, t\right):=P_{t}\left(x^{\prime}\right):=t^{1-n} P\left(x^{\prime} / t\right)$, for each $x \in \mathbb{R}^{n-1}$ and $t>0$, then the function $K=\left(K_{\alpha \beta}\right)_{1 \leq \alpha, \beta \leq M}$ satisfies (in the sense of distributions)

$$
L K_{\cdot \beta}=0 \text { in } \mathbb{R}_{+}^{n} \text { for each } \beta \in\{1, \ldots, M\} \text {. }
$$

Remark 3.2. The following comments pertain to Definition 3.1. 
(i) Condition (a) ensures that the integral in part (b) is absolutely convergent.

(ii) From $(a)$ and $(b)$ one can easily check that for each $p \in(1, \infty]$ there exists a finite constant $C=C(c, M, n, p)>0$ with the property that if $f \in L^{p}\left(\mathbb{R}^{n-1}\right)$ and $u\left(x^{\prime}, t\right):=\left(P_{t} * f\right)\left(x^{\prime}\right)$ for $\left(x^{\prime}, t\right) \in \mathbb{R}_{+}^{n}$, then

$$
\|\mathcal{N} u\|_{L^{p}\left(\partial \mathbb{R}_{+}^{n}\right)} \leq C\|f\|_{L^{p}\left(\mathbb{R}^{n-1}\right)} \text { and }\left.u\right|_{\partial \mathbb{R}_{+}^{n}} ^{n . t .}=f \text { a.e. in } \mathbb{R}^{n-1} \text {. }
$$

(iii) Condition $(c)$ and the ellipticity of the operator $L$ ensure that $K \in \mathscr{C}^{\infty}\left(\mathbb{R}_{+}^{n}\right)$. Given that $P\left(x^{\prime}\right)=K\left(x^{\prime}, 1\right)$ for each point $x^{\prime} \in \mathbb{R}^{n-1}$, we then deduce that $P \in \mathscr{C}^{\infty}\left(\mathbb{R}^{n-1}\right)$. Furthermore, via a direct calculation it may be checked that

$$
\partial_{t}\left[P_{t}\left(x^{\prime}\right)\right]=-\sum_{j=1}^{n-1} \partial_{x_{j}}\left[\frac{x_{j}}{t} P_{t}\left(x^{\prime}\right)\right] \text { for every }\left(x^{\prime}, t\right) \in \mathbb{R}_{+}^{n} .
$$

(iv) Condition (b) is equivalent to $\lim _{t \rightarrow 0^{+}} P_{t}\left(x^{\prime}\right)=\delta_{0^{\prime}}\left(x^{\prime}\right) I_{M \times M}$ in $\mathscr{D}^{\prime}\left(\mathbb{R}^{n-1}\right)$, where $\delta_{0^{\prime}}$ is Dirac's distribution with mass at the origin $0^{\prime}$ of $\mathbb{R}^{n-1}$.

Poisson kernels for elliptic boundary value problems in a half-space have been studied extensively in [1], [2], [6, §10.3], [13], [14], [15]. Here we record a corollary of more general work done by S. Agmon, A. Douglis, and L. Nirenberg in [2].

Theorem 3.3. Any elliptic differential operator L as in (1.1) has a Poisson kernel $P$ in the sense of Definition 3.1, which has the additional property that the function $K\left(x^{\prime}, t\right):=P_{t}\left(x^{\prime}\right)$ for all $\left(x^{\prime}, t\right) \in \mathbb{R}_{+}^{n}$, satisfies $K \in \mathscr{C}^{\infty}\left(\overline{\mathbb{R}_{+}^{n}} \backslash B(0, \varepsilon)\right)$ for every $\varepsilon>0$ and $K(\lambda x)=\lambda^{1-n} K(x)$ for all $x \in \mathbb{R}_{+}^{n}$ and $\lambda>0$.

Hence, in particular, for each $\alpha \in \mathbb{N}_{0}^{n}$ there exists $C_{\alpha} \in(0, \infty)$ with the property that $\left|\left(\partial^{\alpha} K\right)(x)\right| \leq C_{\alpha}|x|^{1-n-|\alpha|}$, for every $x \in \overline{\mathbb{R}_{+}^{n}} \backslash\{0\}$.

One important consequence of the existence of a Poisson kernel $P$ for an operator $L$ in the upper-half space is that for every $f \in L^{p}\left(\mathbb{R}^{n-1}\right)$ the convolution $\left(P_{t} * f\right)\left(x^{\prime}\right)$ for $\left(x^{\prime}, t\right) \in \mathbb{R}_{+}^{n}$, yields a solution for the $L^{p}$-Dirichlet problem for $L$ in the upperhalf space. Hence, the difficulty in proving well-posedness for such a problem comes down to proving uniqueness. In the case of the Laplacian, this is done by employing the maximum principle for harmonic functions, a tool not available in the case of systems. In [8] we overcome this difficulty by constructing an appropriate Green function associated with the $L^{p}$-Dirichlet problem for $L$ in the upper-half space.

Theorem 3.4. [8] For each $p \in(1, \infty)$ the $L^{p}$-Dirichlet boundary value problem for $L$ in $\mathbb{R}_{+}^{n}$, that is, (1.3) with $\ell=0$, has a unique solution $u=\left(u_{\beta}\right)_{1 \leq \beta \leq M}$ satisfying, for some finite $C=C(L, n, p)>0$,

$$
\|\mathcal{N} u\|_{L^{p}\left(\partial \mathbb{R}_{+}^{n}\right)} \leq C\|f\|_{L^{p}\left(\mathbb{R}^{n-1}\right)}
$$

Moreover, the solution $u$ is given by

$$
u\left(x^{\prime}, t\right)=\left(P_{t} * f\right)\left(x^{\prime}\right)=\left(\int_{\mathbb{R}^{n-1}}\left(P_{\beta \alpha}\right)_{t}\left(x^{\prime}-y^{\prime}\right) f_{\alpha}\left(y^{\prime}\right) d y^{\prime}\right)_{\beta}
$$

for all $\left(x^{\prime}, t\right) \in \mathbb{R}_{+}^{n}$, where $P$ is the Poisson kernel from Theorem 3.3.

A corollary of this theorem is the uniqueness of the Poisson kernel for $L$ in $\mathbb{R}_{+}^{n}$.

Proposition 3.5. Any operator $L$ as in (1.1)-(1.2) has a unique Poisson kernel as in Definition 3.1 (which is the Poisson kernel given by Theorem 3.3). 
Proof. Suppose $L$ has two Poisson kernels, say $P$ and $Q$, in $\mathbb{R}_{+}^{n}$. Then for each $p \in(1, \infty)$ and every $f \in L^{p}\left(\mathbb{R}^{n-1}\right)$, the function $u\left(x^{\prime}, t\right):=\left(P_{t}-Q_{t}\right) * f\left(x^{\prime}\right)$ for $\left(x^{\prime}, t\right) \in \mathbb{R}_{+}^{n}$, is a solution of the homogeneous $L^{p}$-Dirichlet boundary value problem in $\mathbb{R}_{+}^{n}$. Hence, by Theorem 3.4, $u=0$ in $\mathbb{R}_{+}^{n}$. This forces $P=Q$ in $\mathbb{R}^{n-1}$.

As mentioned before, there are multiple coefficient tensors which yield a given system $L$ as in (1.1). The following proposition paves the way for singling out, in Definition 3.7 formulated a little later, a special subclass among all these coefficient tensors.

Proposition 3.6. [7] Assume that $A=\left(a_{r s}^{\alpha \beta}\right)_{\substack{1 \leq r, s \leq n \\ 1 \leq \alpha, \beta \leq M}}$ is a coefficient tensor with complex entries satisfying the Legendre-Hadamard ellipticity condition (1.2). Let L be the system associated with the given coefficient tensor $A$ as in (1.1) and denote by $E=\left(E_{\gamma \beta}\right)_{1 \leq \gamma, \beta \leq M}$ the fundamental solution from Theorem 2.1 for the system L. Also, let $\operatorname{Symb}_{L}(\xi):=-\left(\xi_{r} \xi_{s} a_{r s}^{\alpha \beta}\right)_{1 \leq \alpha, \beta \leq M}$, for $\xi \in \mathbb{R}^{n} \backslash\{0\}$, denote the symbol of the differential operator $L$ and set

$$
\left(S_{\gamma \beta}(\xi)\right)_{1 \leq \gamma, \beta \leq M}:=\left[\operatorname{Symb}_{L}(\xi)\right]^{-1} \in \mathbb{C}^{M \times M}, \quad \forall \xi \in \mathbb{R}^{n} \backslash\{0\} .
$$

Then the following two conditions are equivalent.

(a) For each $s, s^{\prime} \in\{1, \ldots, n\}$ and each $\alpha, \gamma \in\{1, \ldots, M\}$ there holds

$$
\left[a_{s^{\prime} s}^{\beta \alpha}-a_{s s^{\prime}}^{\beta \alpha}+\xi_{r} a_{r s}^{\beta \alpha} \partial_{\xi_{s^{\prime}}}-\xi_{r} a_{r s^{\prime}}^{\beta \alpha} \partial_{\xi_{s}}\right] S_{\gamma \beta}(\xi)=0, \quad \forall \xi \in \mathbb{R}^{n} \backslash\{0\},
$$

and (with $\sigma_{S^{1}}$ denoting the arc-length measure on $S^{1}$ )

$$
\int_{S^{1}}\left(a_{r s}^{\beta \alpha} \xi_{s^{\prime}}-a_{r s^{\prime}}^{\beta \alpha} \xi_{s}\right)\left(\xi_{r} S_{\gamma \beta}(\xi)\right) d \sigma_{S^{1}}(\xi)=0 \text { if } n=2 .
$$

(b) There exists a matrix-valued function $k=\left\{k_{\gamma \alpha}\right\}_{1 \leq \gamma, \alpha \leq M}: \mathbb{R}^{n} \backslash\{0\} \rightarrow \mathbb{C}^{M \times M}$ with the property that for each $\gamma, \alpha \in\{1, \ldots, M\}$ and $s \in\{1, \ldots, n\}$ one has

$$
a_{r s}^{\beta \alpha}\left(\partial_{r} E_{\gamma \beta}\right)(x)=x_{s} k_{\gamma \alpha}(x) \text { for all } x \in \mathbb{R}^{n} \backslash\{0\} .
$$

In light of the properties of the fundamental solution, condition (3.9) readily implies that

$$
k \in \mathscr{C}^{\infty}\left(\mathbb{R}^{n} \backslash\{0\}\right) \text { and } k \text { is even and homogeneous of degree }-n \text {. }
$$

Note that condition (a) in Proposition 3.6 is entirely formulated in terms of the coefficient tensor $A$. This suggests making the following definition (recall that $\mathfrak{A}_{L}$ has been introduced in (2.8)).

Definition 3.7. Given a second-order elliptic system $L$ with constant complex coefficients as in (1.1)-(1.2), call a coefficient tensor

$$
A=\left(a_{r s}^{\alpha \beta}\right)_{\substack{1 \leq r, s \leq n \\ 1 \leq \alpha, \beta \leq M}} \in \mathfrak{A}_{L}
$$

distinguished provided condition (a) in Proposition 3.6 holds, and denote by $\mathfrak{A}_{L}^{\text {dis }}$ the totality of such distinguished coefficient tensors for L, i.e.,

$$
\mathfrak{A}_{L}^{\text {dis }}:=\left\{A=\left(a_{r s}^{\alpha \beta}\right)_{\substack{1 \leq r, s \leq n \\ 1 \leq \alpha, \beta \leq M}} \in \mathfrak{A}_{L}\right. \text { : conditions (3.7)-(3.8) hold for each }
$$

$$
\left.s, s^{\prime} \in\{1, \ldots, n\} \text { and } \alpha, \gamma \in\{1, \ldots, M\}\right\} .
$$


Remark 3.8. We claim that $\mathfrak{A}_{L}^{\text {dis }} \neq \emptyset$ whenever $M=1$. More specifically, when $M=1$, i.e., $L=\operatorname{div} A \nabla$ with $A=\left(a_{r s}\right)_{1 \leq r, s \leq n} \in \mathbb{C}^{n \times n}$, one has $A_{\text {sym }} \in \mathfrak{A}_{L}^{\text {dis }}$. To see that this is the case, recall that checking the membership of $A_{\text {sym }}$ to $\mathfrak{A}_{L}^{\text {dis }}$ comes down to verifying conditions (3.7)-(3.8) for the entries in the matrix $A_{\text {sym }}$. Note that for each index $s \in\{1, \ldots, n\}$ we have in this case

$$
\partial_{\xi_{s}}\left[\operatorname{Symb}_{L}(\xi)\right]^{-1}=2\left[\operatorname{Symb}_{L}(\xi)\right]^{-2}\left(A_{\mathrm{sym}} \xi\right)_{s}, \quad \forall \xi \in \mathbb{R}^{n} \backslash\{0\},
$$

and (3.7) readily follows from this. Moreover, if $n=2$, condition (3.8) reduces to checking that

$$
\int_{S^{1}} \frac{\left(A_{\mathrm{sym}} \xi\right) \cdot\left(\xi_{2},-\xi_{1}\right)}{\left(A_{\mathrm{sym}} \xi\right) \cdot \xi} d \sigma_{S^{1}}(\xi)=0
$$

The key observation in this regard is that if $f(\theta):=\left.\left[\left(A_{\operatorname{sym}} \xi\right) \cdot \xi\right]\right|_{\xi=(\cos \theta, \sin \theta)}$ then

$$
\left.\frac{\left(A_{\mathrm{sym}} \xi\right) \cdot\left(\xi_{2},-\xi_{1}\right)}{\left(A_{\mathrm{sym}} \xi\right) \cdot \xi}\right|_{\xi=(\cos \theta, \sin \theta)}=-\frac{f^{\prime}(\theta)}{2 f(\theta)}, \quad \forall \theta \in(0,2 \pi) .
$$

Now (3.14) readily follows from (3.15), proving that indeed $A_{\text {sym }} \in \mathfrak{A}_{L}^{\text {dis }}$.

One of the main features of elliptic systems having a distinguished coefficient tensor is that their Poisson kernels have a special form. This is made more precise in the next proposition.

Proposition 3.9. [8] Let $L$ be a constant coefficient system as in (1.1)-(1.2). Assume that $\mathfrak{A}_{L}^{\text {dis }} \neq \emptyset$ and let $k=\left\{k_{\gamma \alpha}\right\}_{1 \leq \gamma, \alpha \leq M}: \mathbb{R}^{n} \backslash\{0\} \rightarrow \mathbb{C}^{M \times M}$ be the function appearing in condition (b) of Proposition 3.6. Then the unique Poisson kernel for $L$ in $\mathbb{R}_{+}^{n}$ from Theorem 3.3 has the form

$$
P\left(x^{\prime}\right)=2 k\left(x^{\prime}, 1\right), \quad \forall x^{\prime} \in \mathbb{R}^{n-1} .
$$

\section{The Dirichlet Problem With DATA In Higher ORDER Sobolev SPACES}

The main result of our paper is the following theorem giving the well-posedness of the Dirichlet boundary value problem in $\mathbb{R}_{+}^{n}$ with data in higher-order Sobolev spaces for constant (complex) coefficient elliptic systems possessing a distinguished coefficient tensor.

Theorem 4.1. Let $L$ be an operator as in (1.1)-(1.2) with the property that $\mathfrak{A}_{L}^{\text {dis } \neq}$ $\emptyset$, and fix $p \in(1, \infty)$ and $\ell \in \mathbb{N}_{0}$. Then the $\ell$-th order Dirichlet boundary value problem for $L$ in $\mathbb{R}_{+}^{n}$,

$$
\left\{\begin{array}{l}
L u=0 \text { in } \mathbb{R}_{+}^{n}, \\
\mathcal{N}\left(\nabla^{k} u\right) \in L^{p}\left(\partial \mathbb{R}_{+}^{n}\right), 0 \leq k \leq \ell \\
\left.u\right|_{\partial \mathbb{R}_{+}^{n}} ^{n . t .}=f \in L_{\ell}^{p}\left(\mathbb{R}^{n-1}\right),
\end{array}\right.
$$

has a unique solution. Moreover, the solution $u$ of (4.1) is given by

$$
u\left(x^{\prime}, t\right)=\left(P_{t} * f\right)\left(x^{\prime}\right), \quad \forall\left(x^{\prime}, t\right) \in \mathbb{R}_{+}^{n},
$$


where $P$ is the Poisson kernel for $L$ in $\mathbb{R}_{+}^{n}$ from Theorem 3.3. Furthermore, there exists a constant $C=C(n, p, L, \ell) \in(0, \infty)$ with the property that

$$
\sum_{k=0}^{\ell}\left\|\mathcal{N}\left(\nabla^{k} u\right)\right\|_{L^{p}\left(\partial \mathbb{R}_{+}^{n}\right)} \leq C\|f\|_{L_{\ell}^{p}\left(\mathbb{R}^{n-1}\right)} .
$$

The remainder of this section is devoted to providing a proof for Theorem 4.1. This requires developing a number of tools, which are introduced and studied first.

To fix notation let $\nabla_{x^{\prime}}:=\left(\partial_{1}, \ldots, \partial_{n-1}\right)$ and, alternatively, use $\partial_{t}$ in place of $\partial_{n}$ if the description $\left(x^{\prime}, t\right)$ of points in $\mathbb{R}^{n-1} \times(0, \infty)$ is emphasized in place of $x \in \mathbb{R}_{+}^{n}$. Also fix $p \in(1, \infty), \ell \in \mathbb{N}$, and let $f \in L_{\ell}^{p}\left(\mathbb{R}^{n-1}\right)$. In view of Theorem 3.4, proving Theorem 4.1 reduces to showing that the function $u\left(x^{\prime}, t\right)=\left(P_{t} * f\right)\left(x^{\prime}\right)$ for $\left(x^{\prime}, t\right) \in \mathbb{R}_{+}^{n}$ satisfies $\mathcal{N}\left(\nabla^{k} u\right) \in L^{p}\left(\partial \mathbb{R}_{+}^{n}\right)$ for $k=1, \ldots, \ell$, as well as (4.3). Suppose $\alpha=\left(\alpha_{1}, \ldots, \alpha_{n}\right) \in \mathbb{N}_{0}$ is such that $|\alpha| \leq \ell$. It is immediate that if $\alpha_{n}=0$ then $\partial^{\alpha} u\left(x^{\prime}, t\right)=\left(P_{t} *\left(\partial^{\alpha} f\right)\right)\left(x^{\prime}\right)$ for $\left(x^{\prime}, t\right) \in \mathbb{R}_{+}^{n}$. The crux of the matter is handling $\partial^{\alpha} u$ when $\alpha_{n} \neq 0$. As you will see below, the special format of the Poisson kernel guaranteed by Proposition 3.9 allows us to prove a set of basic identities expressing $\partial_{t}^{k}\left[\left(P_{t} * f\right)\left(x^{\prime}\right)\right]$ as a linear combination of $\left(P_{t} * \nabla_{x^{\prime}}^{k} f\right)\left(x^{\prime}\right)$ and convolutions of certain auxiliary kernels with derivatives of $f$. Here is the class of auxiliary kernels just alluded to.

Definition 4.2. Given an operator $L$ as in (1.1)-(1.2) denote by $E$ the fundamental solution for $L$ from Theorem 2.1. Then for each $j \in\{1, \ldots, n\}$ define the auxiliary matrix-valued kernel function

$$
Q^{(j)}\left(x^{\prime}\right):=\left(Q_{\alpha \beta}^{(j)}\left(x^{\prime}\right)\right)_{1 \leq \alpha, \beta \leq M}:=\left(\left(\partial_{j} E_{\alpha \beta}\right)\left(x^{\prime}, 1\right)\right)_{1 \leq \alpha, \beta \leq M}, \quad \forall x^{\prime} \in \mathbb{R}^{n-1} .
$$

In the next lemma we describe some of the basic properties of the auxiliary kernels just introduced.

Lemma 4.3. Let $L$ be an operator as in (1.1)-(1.2) and let $\left\{Q_{\alpha \beta}^{(j)}\right\}_{j, \alpha, \beta}$ be the family of functions from (4.4). Then the following are true.

(a) There exists some constant $C=C(n, L) \in(0, \infty)$ such that for each indices $j \in\{1, \ldots, n\}$ and $\alpha, \beta \in\{1, \ldots, M\}$ one has

$$
Q_{\alpha \beta}^{(j)} \in \mathscr{C}^{\infty}\left(\mathbb{R}^{n-1}\right) \text { and }\left|Q_{\alpha \beta}^{(j)}\left(x^{\prime}\right)\right| \leq \frac{C}{\left(\left|x^{\prime}\right|+1\right)^{n-1}} \quad \forall x^{\prime} \in \mathbb{R}^{n-1} .
$$

(b) For each $j, r \in\{1, \ldots, n\}$ and every $\alpha, \gamma \in\{1, \ldots, M\}$ we have

$$
\partial_{j}\left[\left(Q_{\alpha \gamma}^{(r)}\right)_{t}\left(x^{\prime}\right)\right]=\partial_{r}\left[\left(Q_{\alpha \gamma}^{(j)}\right)_{t}\left(x^{\prime}\right)\right], \quad \forall\left(x^{\prime}, t\right) \in \mathbb{R}_{+}^{n} .
$$

(c) Given any $f \in L^{p}\left(\mathbb{R}^{n-1}\right)$ where $p \in(1, \infty)$, along with $j \in\{1, \ldots, n\}$ and $\alpha, \beta \in\{1, \ldots, M\}$, define the function

$$
u_{\alpha \beta}^{(j)}: \mathbb{R}_{+}^{n} \rightarrow \mathbb{C}, \quad u_{\alpha \beta}^{(j)}\left(x^{\prime}, t\right):=\left[\left(Q_{\alpha \beta}^{(j)}\right)_{t} * f\right]\left(x^{\prime}\right), \quad \forall\left(x^{\prime}, t\right) \in \mathbb{R}_{+}^{n} .
$$

Then there exists a constant $C \in(0, \infty)$ independent of $f$ such that

$$
\left\|\mathcal{N} u_{\alpha \beta}^{(j)}\right\|_{L^{p}\left(\mathbb{R}^{n-1}\right)} \leq C\|f\|_{L^{p}\left(\mathbb{R}^{n-1}\right)} .
$$

Proof. Let $E$ be the fundamental solution for $L$ defined in Theorem 2.1. The fact that the claims in (a) hold is a consequence of (4.4), and Theorem 2.1 parts $(a)$ and 
(d). Next, fix $j \in\{1, \ldots, n\}, \alpha, \beta \in\{1, \ldots, M\}$ and let $\left(x^{\prime}, t\right) \in \mathbb{R}_{+}^{n}$. Since $\nabla E$ is positive homogeneous of order $1-n$ in $\mathbb{R}^{n} \backslash\{0\}$ (cf. property (c) in Theorem 2.1), one has

$$
\left(Q_{\gamma \beta}^{(r)}\right)_{t}\left(x^{\prime}\right)=t^{1-n}\left(\partial_{r} E_{\gamma \beta}\right)\left(x^{\prime} / t, 1\right)=\left(\partial_{r} E_{\gamma \beta}\right)\left(x^{\prime}, t\right), \quad \forall r \in\{1, \ldots, n\} .
$$

Now (4.9) and the first condition in (4.5) imply that for every $j, r \in\{1, \ldots, n\}$,

$$
\partial_{j}\left[\left(Q_{\alpha \gamma}^{(r)}\right)_{t}\left(x^{\prime}\right)\right]=\left(\partial_{j} \partial_{r} E_{\gamma \beta}\right)\left(x^{\prime}, t\right)=\left(\partial_{r} \partial_{j} E_{\gamma \beta}\right)\left(x^{\prime}, t\right)=\partial_{r}\left[\left(Q_{\alpha \gamma}^{(j)}\right)_{t}\left(x^{\prime}\right)\right],
$$

proving (4.6).

There remains to prove the claim in $(c)$. To this end, let $f \in L^{p}\left(\mathbb{R}^{n-1}\right)$ for some $p \in(1, \infty)$. Then by $(4.7)$ and $(4.9)$ we have

$$
u_{\alpha \beta}^{(j)}\left(x^{\prime}, t\right)=\int_{\mathbb{R}^{n-1}}\left(\partial_{j} E_{\alpha \beta}\right)\left(x^{\prime}-y^{\prime}, t\right) f\left(y^{\prime}\right) d y^{\prime}, \quad \forall\left(x^{\prime}, t\right) \in \mathbb{R}_{+}^{n} .
$$

If we now write $K=\partial_{j} E_{\alpha \beta}$, the properties of $E$ (cf. Theorem 2.1) imply that $K \in \mathscr{C}^{\infty}\left(\mathbb{R}^{n} \backslash\{0\}\right)$ with $K(-x)=-K(x)$ and $K(\lambda x)=\lambda^{-(n-1)} K(x)$ for every $\lambda>0$ and $x \in \mathbb{R}^{n} \backslash\{0\}$. We can therefore invoke standard Calderón-Zygmund theory and conclude that (4.8) holds.

In order to elaborate on the relationship between the family of auxiliary kernels from Definition 4.2 and the Poisson kernel for the operator $L$ in $\mathbb{R}_{+}^{n}$, under the assumption $\mathfrak{A}_{L}^{\text {dis }} \neq \emptyset$, we first need to introduce some notation which facilitates the subsequent discussion. Specifically, given a coefficient tensor $A=\left(a_{r s}^{\alpha \beta}\right)_{r, s, \alpha, \beta}$ with complex entries satisfying the Legendre-Hadamard ellipticity condition (1.2), for each $r, s \in\{1, \ldots, n\}$ abbreviate

$$
A_{r s}:=\left(a_{r s}^{\alpha \beta}\right)_{1 \leq \alpha, \beta \leq M} .
$$

Note that the ellipticity condition (1.2) written for $\xi:=\mathbf{e}_{n} \in \mathbb{R}^{n}$ yields, in particular, that $A_{n n}=\left(a_{n n}^{\alpha \beta}\right)_{1 \leq \alpha, \beta \leq M} \in \mathbb{C}^{M \times M}$ is an invertible matrix. Next, for each sufficiently smooth vector field $u=\left(u_{\beta}\right)_{1 \leq \beta \leq M}$, define

$$
D_{A} u:=\left(a_{n s}^{\alpha \beta} \partial_{s} u_{\beta}\right)_{1 \leq \alpha \leq M}
$$

and set (with the superscript $\top$ denoting transposition)

$$
\partial_{\tan } u:=-\left(A_{n n}^{\top}\right)^{-1}\left[\left(\sum_{s=1}^{n-1} a_{s n}^{\beta \alpha} \partial_{s} u_{\beta}\right)_{1 \leq \alpha \leq M}\right] .
$$

The notation $\partial_{\tan }$ is justified by the fact that its expression only involves partial derivatives in directions tangent to the boundary of the upper-half space $\partial \mathbb{R}_{+}^{n}$.

For reasons that will become clear momentarily, we are interested in decomposing the operator $\partial_{t}\left(=\partial_{n}\right)$ as the sum between a linear combination of the partial derivative operators $\partial_{j}, j=1, \ldots, n-1$, (which correspond to tangential directions to $\partial \mathbb{R}_{+}^{n}$ ) and a suitable (matrix) multiple of $D_{A^{\top}}$.

Lemma 4.4. One has $\partial_{t}=\partial_{\tan }+\left(A_{n n}^{\top}\right)^{-1} D_{A^{\top}}$. 
Proof. Given $u=\left(u_{\beta}\right)_{1 \leq \beta \leq M} \in \mathscr{C}^{1}\left(\mathbb{R}_{+}^{n}\right)$ we may write

$$
\begin{aligned}
\partial_{t} u-\left(A_{n n}^{\top}\right)^{-1} D_{A^{\top}} u & =\left(A_{n n}^{\top}\right)^{-1}\left[A_{n n}^{\top} \partial_{t} u-D_{A^{\top}} u\right] \\
& =\left(A_{n n}^{\top}\right)^{-1}\left[\left(a_{n n}^{\beta \alpha} \partial_{t} u_{\beta}-a_{s n}^{\beta \alpha} \partial_{s} u_{\beta}\right)_{1 \leq \alpha \leq M}\right] \\
& =-\left(A_{n n}^{\top}\right)^{-1}\left[\left(\sum_{s=1}^{n-1} a_{s n}^{\beta \alpha} \partial_{s} u_{\beta}\right)_{1 \leq \alpha \leq M}\right]=\partial_{\tan } u,
\end{aligned}
$$

as desired.

We are now ready to state and prove a number of basic identities relating the family of auxiliary kernels from Definition 4.2 to the Poisson kernel for the operator $L$, under the assumption that the latter has a distinguished coefficient tensor.

Proposition 4.5. Let $L$ be an operator as in (1.1)-(1.2) with the property that $\mathfrak{A}_{L}^{\text {dis }} \neq \emptyset$. Denote by $P$ the Poisson kernel for $L$ from Theorem 3.3 and fix some coefficient tensor

$$
A=\left(a_{r s}^{\alpha \beta}\right)_{\substack{1 \leq r, s \leq n \\ 1 \leq \alpha, \beta \leq M}} \in \mathfrak{A}_{L}^{\text {dis }} .
$$

Then the auxiliary kernels $\left\{Q_{\alpha \beta}^{(j)}\right\}_{j, \alpha, \beta}$ introduced in Definition 4.2 satisfy the following properties:

(a) for each $\alpha, \gamma \in\{1, \ldots, M\}$ one has for every $x^{\prime} \in \mathbb{R}^{n-1}$ and every $t=x_{n}>0$

$$
2 a_{r s}^{\beta \alpha}\left(Q_{\gamma \beta}^{(r)}\right)_{t}\left(x^{\prime}\right)=\frac{x_{s}}{t}\left(P_{\gamma \alpha}\right)_{t}\left(x^{\prime}\right) \text { for each } s \in\{1, \ldots, n\} ;
$$

(b) for every $\alpha, \gamma \in\{1, \ldots, M\}$ one has for every $x^{\prime} \in \mathbb{R}^{n-1}$ and every $t>0$

$$
\partial_{t}\left[\left(P_{\gamma \alpha}\right)_{t}\left(x^{\prime}\right)\right]=-2 \sum_{s=1}^{n-1} a_{r s}^{\beta \alpha} \partial_{s}\left[\left(Q_{\gamma \beta}^{(r)}\right)_{t}\left(x^{\prime}\right)\right]
$$

(c) for each $\gamma \in\{1, \ldots, M\}$ one has

$$
\begin{aligned}
\left(Q_{\gamma \alpha}^{(n)}\right)_{1 \leq \alpha \leq M}= & \frac{1}{2}\left(A_{n n}^{\top}\right)^{-1}\left(\left(P_{\gamma \mu}\right)_{1 \leq \mu \leq M}\right) \\
& -\sum_{s=1}^{n-1}\left(A_{n n}^{\top}\right)^{-1}\left(\left(a_{s n}^{\beta \mu} Q_{\gamma \beta}^{(s)}\right)_{1 \leq \mu \leq M}\right) \text { in } \mathbb{R}^{n-1} .
\end{aligned}
$$

Proof. Since $\mathfrak{A}_{L}^{\text {dis }} \neq \emptyset$, Proposition 3.6 ensures that the Poisson kernel $P$ satisfies (3.16). Hence, if $E$ is the fundamental solution for $L$ from Theorem 2.1, starting with (4.4), then using (3.9), and then (3.16), for each $s \in\{1, \ldots, n\}$, $\alpha, \gamma \in\{1, \ldots, M\}$, for every $x^{\prime} \in \mathbb{R}^{n-1}$ and $t=x_{n}>0$ we obtain

$$
\begin{aligned}
2 a_{r s}^{\beta \alpha}\left(Q_{\gamma \beta}^{(r)}\right)_{t}\left(x^{\prime}\right) & =2 a_{r s}^{\beta \alpha} t^{1-n}\left(\partial_{r} E_{\gamma \beta}\right)\left(x^{\prime} / t, 1\right) \\
& =2 t^{1-n}\left(x^{\prime} / t, 1\right)_{s} k_{\gamma \alpha}\left(x^{\prime} / t, 1\right) \\
& =\left(x^{\prime} / t, 1\right)_{s}\left(P_{\gamma \alpha}\right)_{t}\left(x^{\prime}\right)=\frac{x_{s}}{t}\left(P_{\gamma \alpha}\right)_{t}\left(x^{\prime}\right) .
\end{aligned}
$$


This takes care of (4.17). The statement in $(b)$ is obtained from (3.3) and (4.17) by writing for every $x^{\prime} \in \mathbb{R}^{n-1}$ and $t>0$

$$
\partial_{t}\left[\left(P_{\gamma \alpha}\right)_{t}\left(x^{\prime}\right)\right]=-\sum_{s=1}^{n-1} \partial_{s}\left[\frac{x_{s}}{t}\left(P_{\gamma \alpha}\right)_{t}\left(x^{\prime}\right)\right]=-2 \sum_{s=1}^{n-1} a_{r s}^{\beta \alpha} \partial_{s}\left[\left(Q_{\gamma \beta}^{(r)}\right)_{t}\left(x^{\prime}\right)\right] .
$$

The next task is to prove (4.19). Recalling (4.4), the term in the left hand-side of (4.19) evaluated at an arbitrary point $x^{\prime} \in \mathbb{R}^{n-1}$ becomes

$$
\begin{aligned}
Q_{\gamma \cdot}^{(n)}\left(x^{\prime}\right) & =\left(\partial_{t} E_{\gamma \cdot}\right)\left(x^{\prime}, 1\right)=\left.\left[\partial_{t} E_{\gamma \cdot} \cdot\left(x^{\prime}, t\right)\right]\right|_{t=1} \\
& =-\sum_{s=1}^{n-1}\left(A_{n n}^{\top}\right)^{-1}\left[a_{s n}^{\beta \cdot}\left(\partial_{s} E_{\gamma \beta}\right)\left(x^{\prime}, 1\right)\right]+\left.\left(A_{n n}^{\top}\right)^{-1}\left[D_{A^{\top}} E_{\gamma \cdot} \cdot\left(x^{\prime}, t\right)\right]\right|_{t=1} \\
& =-\sum_{s=1}^{n-1}\left(A_{n n}^{\top}\right)^{-1}\left[a_{s n}^{\beta \cdot} Q_{\gamma \beta}^{(s)}\left(x^{\prime}\right)\right]+\left(A_{n n}^{\top}\right)^{-1}\left[a_{j n}^{\beta \cdot} Q_{\gamma \beta}^{(j)}\left(x^{\prime}\right)\right] \\
& =-\sum_{s=1}^{n-1}\left(A_{n n}^{\top}\right)^{-1}\left[a_{s n}^{\beta \cdot} Q_{\gamma \beta}^{(s)}\left(x^{\prime}\right)\right]+\frac{1}{2}\left(A_{n n}^{\top}\right)^{-1}\left[P_{\gamma \cdot}\left(x^{\prime}\right)\right] .
\end{aligned}
$$

The third equality in (4.22) uses the decomposition of $\partial_{t}$ as in Lemma 4.4 and (4.13), the forth equality is based on (4.4) and (4.13), while the last equality is a consequence of (4.17) specialized to the case when $s=n$.

It is useful to rephrase the kernel identities from Proposition 4.5 in terms of their associated convolution operators. Before doing so, the reader is advised to recall the piece of notation introduced in (4.12).

Proposition 4.6. Let $L$ be an operator as in (1.1)-(1.2) with the property that $\mathfrak{A}_{L}^{\text {dis }} \neq \emptyset$. Denote by $P$ the Poisson kernel for $L$ from Theorem 3.3, and fix some coefficient tensor

$$
A=\left(a_{r s}^{\alpha \beta}\right)_{\substack{1 \leq r, s \leq n \\ 1 \leq \alpha \leq M}} \in \mathfrak{A}_{L}^{d i s}
$$

Consider the family of auxiliary kernels $\left\{Q_{\alpha \beta}^{(j)}\right\}_{j, \alpha, \beta}$ introduced in Definition 4.2 and let $p \in(1, \infty)$. Then, for every $t>0$, the following identities hold:

(a) for every $f=\left(f_{\alpha}\right)_{\alpha} \in L^{p}\left(\mathbb{R}^{n-1}\right)$ one has

$$
Q_{t}^{(n)} * f=\frac{1}{2} P_{t} * A_{n n}^{-1} f-\sum_{s=1}^{n-1} Q_{t}^{(s)} * A_{s n} A_{n n}^{-1} f \quad \text { in } \mathbb{R}^{n-1} ;
$$

(b) if $f=\left(f_{\alpha}\right)_{\alpha} \in L_{1}^{p}\left(\mathbb{R}^{n-1}\right)$, then for each $\gamma \in\{1, \ldots, M\}$,

$$
\partial_{t}\left[\left(P_{t} * f\right)_{\gamma}\right]=-2 \sum_{s=1}^{n-1} a_{r s}^{\beta \alpha}\left(\left(Q_{\gamma \beta}^{(r)}\right)_{t} * \partial_{s} f_{\alpha}\right) \quad \text { in } \mathbb{R}^{n-1},
$$

and for every $r \in\{1, \ldots, n-1\}$,

$$
\partial_{t}\left[\left(Q_{t}^{(r)} * f\right)_{\gamma}\right]=\left(Q_{t}^{(n)} *\left(\partial_{r} f\right)\right)_{\gamma} \quad \text { in } \mathbb{R}^{n-1} .
$$


Proof. Fix $f=\left(f_{\alpha}\right)_{\alpha} \in L^{p}\left(\mathbb{R}^{n-1}\right)$ and $\gamma \in\{1, \ldots, M\}$. To obtain (4.24), we convolve (4.19) with $f$ in order to write

$$
\begin{aligned}
\left(Q_{t}^{(n)} * f\right)_{\gamma} & =\left(Q_{\gamma \alpha}^{(n)}\right)_{t} * f_{\alpha} \\
& =\frac{1}{2}\left(\left(A_{n n}^{\top}\right)^{-1}\right)_{\alpha \mu}\left(P_{\gamma \mu}\right)_{t} * f_{\alpha}-\sum_{s=1}^{n-1}\left(\left(A_{n n}^{\top}\right)^{-1}\right)_{\alpha \mu} a_{s n}^{\beta \mu}\left(Q_{\gamma \beta}^{(s)}\right)_{t} * f_{\alpha} \\
& =\frac{1}{2}\left(P_{\gamma \mu}\right)_{t} *\left(A_{n n}^{-1} f\right)_{\mu}-\sum_{s=1}^{n-1} a_{s n}^{\beta \mu}\left(Q_{\gamma \beta}^{(s)}\right)_{t} *\left(A_{n n}^{-1} f\right)_{\mu} \\
& =\frac{1}{2}\left(P_{t} * A_{n n}^{-1} f\right)_{\gamma}-\sum_{s=1}^{n-1}\left(Q_{t}^{(s)} * A_{s n} A_{n n}^{-1} f\right)_{\gamma} \text { in } \mathbb{R}^{n-1} .
\end{aligned}
$$

Moving on, suppose that actually $f \in L_{1}^{p}\left(\mathbb{R}^{n-1}\right)$ and let $x^{\prime} \in \mathbb{R}^{n-1}$ be arbitrary. Then we have

$$
\begin{aligned}
\partial_{t}\left[\left(P_{t} * f\right)_{\gamma}\left(x^{\prime}\right)\right] & =\int_{\mathbb{R}^{n-1}} \partial_{t}\left[\left(P_{\gamma \mu}\right)_{t}\left(x^{\prime}-y^{\prime}\right)\right] f_{\mu}\left(y^{\prime}\right) d y^{\prime} \\
& =-2 \sum_{s=1}^{n-1} a_{r s}^{\beta \mu} \int_{\mathbb{R}^{n-1}} \partial_{x_{s}}\left[\left(Q_{\gamma \beta}^{(r)}\right)_{t}\left(x^{\prime}-y^{\prime}\right)\right] f_{\mu}\left(y^{\prime}\right) d y^{\prime} \\
& =-2 \sum_{s=1}^{n-1} a_{r s}^{\beta \mu}\left(\left(Q_{\gamma \beta}^{(r)}\right)_{t} * \partial_{s} f_{\mu}\right)\left(x^{\prime}\right)
\end{aligned}
$$

where in the second equality in (4.28) we have employed (4.18). This proves (4.25). We are left with justifying (4.26). If $r \in\{1, \ldots, n-1\}$, then making use of (4.6) with $j=n$ allows us to write

$$
\begin{aligned}
\partial_{t}\left[\left(Q_{t}^{(r)} * f\right)_{\gamma}\right] & =\partial_{t}\left[\left(Q_{\gamma \alpha}^{(r)}\right)_{t} * f_{\alpha}\right]=\partial_{r}\left[\left(Q_{\gamma \alpha}^{(n)}\right)_{t} * f_{\alpha}\right] \\
& =\left(Q_{\gamma \alpha}^{(n)}\right)_{t} *\left(\partial_{r} f_{\alpha}\right)=\left(Q_{t}^{(n)} *\left(\partial_{r} f\right)\right)_{\gamma} \text { in } \mathbb{R}^{n-1}
\end{aligned}
$$

The proof of the proposition is therefore finished.

The following convention is designed to facilitate the remaining portion of the exposition in this section.

Convention 4.7. Given two vectors $f$ and $g$, we will use the notation $f \equiv g$ to indicate that each component of $f$ may be written as a finite linear combination of the components of $g$. Also, given a coefficient tensor $A=\left(a_{j k}^{\alpha \beta}\right)_{\alpha, \beta, j, k}$, the notation $M_{A} f$ is used to indicate that some (or all) of the components of the vector $f$ are multiplied with entries from $A$, or from $\left(A_{n n}\right)^{-1}$. By $\partial_{\tau}$ we denote any of the derivatives $\partial_{1}, \ldots, \partial_{n-1}$, and write $\partial_{\tau}^{k}$ for its $k$-fold iteration. Finally, concerning the kernels from (4.4), we agree that $Q^{\mathrm{I}}$ denotes any $M \times M$ matrix with entries of the form $Q_{\alpha \beta}^{(s)}$ where $s \in\{1, \ldots, n-1\}$ and $\alpha, \beta \in\{1, \ldots, M\}$. On the other hand, $Q^{\mathrm{II}}$ denotes any $M \times M$ matrix with entries of the form $Q_{\alpha \beta}^{(n)}$ where $\alpha, \beta \in\{1, \ldots, M\}$.

Convention 4.7 may now be used to succinctly summarize the identities in Proposition 4.6, as follows. 
Proposition 4.8. Retain the hypotheses from Proposition 4.6. Then the properties listed below (formulated using Convention 4.7) are true for every $t>0$.

(a) If $f \in L^{p}\left(\mathbb{R}^{n-1}\right)$, then

$$
Q_{t}^{\mathrm{II}} * f \equiv P_{t} * M_{A} f+Q_{t}^{\mathrm{I}} * M_{A} f \quad \text { in } \mathbb{R}^{n-1} .
$$

(b) If $f \in L_{1}^{p}\left(\mathbb{R}^{n-1}\right)$, then pointwise in $\mathbb{R}^{n-1}$ one has

$$
\begin{aligned}
\partial_{t}\left[Q_{t}^{\mathrm{I}} * f\right] & \equiv Q_{t}^{\mathrm{II}} * \partial_{\tau} f \equiv P_{t} *\left(M_{A} \partial_{\tau} f\right)+Q_{t}^{\mathrm{I}} *\left(M_{A} \partial_{\tau} f\right) \\
\partial_{t}\left[P_{t} * f\right] & \equiv M_{A} Q_{t}^{\mathrm{I}} * \partial_{\tau} f+M_{A} Q_{t}^{\mathrm{II}} * \partial_{\tau} f \\
& \equiv M_{A} Q_{t}^{\mathrm{I}} *\left(M_{A} \partial_{\tau} f\right)+M_{A} P_{t} *\left(M_{A} \partial_{\tau} f\right) .
\end{aligned}
$$

Proof. Identity (4.31) is a condensed version of (4.24). The first part in (4.32) is a rewriting of (4.26), while the second part is a consequence of (4.31). The first part in (4.33) abbreviates (4.25), while the last part follows from the first part and (4.31).

We are now in a position to formulate our main identities pertaining to higher order derivatives of the operator of convolution with the Poisson kernel under the assumption that the differential operator $L$ has a distinguished coefficient tensor.

Proposition 4.9. Let $L$ be an operator as in (1.1)-(1.2) with the property that $\mathfrak{A}_{L}^{\text {dis }} \neq \emptyset$. Fix some coefficient tensor $A \in \mathfrak{A}_{L}^{\text {dis }}$ and denote by $P$ the Poisson kernel for $L$ from Theorem 3.3. Also, let $p \in(1, \infty), k \in \mathbb{N}_{0}$, and for some $f \in L_{k}^{p}\left(\mathbb{R}^{n-1}\right)$ define the function

$$
u\left(x^{\prime}, t\right):=\left(P_{t} * f\right)\left(x^{\prime}\right), \quad \forall\left(x^{\prime}, t\right) \in \mathbb{R}_{+}^{n} .
$$

Then, for every $\left(x^{\prime}, t\right) \in \mathbb{R}_{+}^{n}$ the following identity (formulated using Convention 4.7) holds:

$$
\nabla^{k} u\left(x^{\prime}, t\right) \equiv M_{A}\left(P_{t} *\left(M_{A} \partial_{\tau}^{k} f\right)\right)\left(x^{\prime}\right)+M_{A}\left(Q_{t}^{\mathrm{I}} *\left(M_{A} \partial_{\tau}^{k} f\right)\right)\left(x^{\prime}\right) .
$$

Proof. Identity (4.35) follows by induction on $k$ from identities (4.32), (4.33) and the fact that for each $\ell \in \mathbb{N}$ and each $t>0$, we have

$$
\partial_{\tau}^{\ell}\left(P_{t} * g\right)=P_{t} * \partial_{\tau}^{\ell} g \quad \text { and } \quad \partial_{\tau}^{\ell}\left(Q_{t}^{\mathrm{I}} * g\right)=Q_{t}^{\mathrm{I}} * \partial_{\tau}^{\ell} g \quad \text { in } \quad \mathbb{R}^{n-1},
$$

for every $g \in L_{\ell}^{p}\left(\mathbb{R}^{n-1}\right)$.

All the ingredients are now in place to proceed with the proof our main result.

Proof of Theorem 4.1. Fix $p \in(1, \infty), \ell \in \mathbb{N}_{0}$, and $f \in L_{\ell}^{p}\left(\mathbb{R}^{n-1}\right)$. The fact that $u$ defined as in (4.2) satisfies the first and last conditions in (4.1) is a consequence of (3.1) and (3.2). In addition, uniqueness for (4.1) is a consequence of Theorem 3.4. Finally, from (4.35), (4.8), and the estimate in (3.2), we deduce that the function (4.2) also satisfies (4.3).

\section{Examples of Boundary PROBlems of mathematical Physics}

In this section we present some examples involving differential operators of basic importance in mathematical physics. For a more detailed discussion (as well as a broader perspective) in this regard, the interested reader is referred to [8]. 
5.1. Scalar second order elliptic equations. Assume that the $n \times n$ matrix $A=\left(a_{r s}\right)_{r, s} \in \mathbb{C}^{n \times n}$ with complex entries satisfies the ellipticity condition

$$
\inf _{\xi \in S^{n-1}} \operatorname{Re}\left[a_{r s} \xi_{r} \xi_{s}\right]>0
$$

and consider the elliptic differential operator $L=\operatorname{div} A \nabla$ in $\mathbb{R}_{+}^{n}$. From Remark 3.8 we know that $\mathfrak{A}_{L}^{d i s} \neq \emptyset$ and, in fact, $A_{\text {sym }} \in \mathfrak{A}_{L}^{\text {dis }}$. Keeping this in mind, Proposition $3.9,(2.15)$, and (3.9), eventually give that

$$
P\left(x^{\prime}\right):=\frac{2}{\omega_{n-1} \sqrt{\operatorname{det}\left(A_{\mathrm{sym}}\right)}} \frac{1}{\left\langle\left(A_{\mathrm{sym}}\right)^{-1}\left(x^{\prime}, 1\right),\left(x^{\prime}, 1\right)\right\rangle^{\frac{n}{2}}}, \quad \forall x^{\prime} \in \mathbb{R}^{n-1},
$$

is the (unique, by Proposition 3.5) Poisson kernel for the operator $L=\operatorname{div} A \nabla$ in $\mathbb{R}_{+}^{n}$. It is reassuring to observe that (5.2) reduces precisely to (1.4) in the case when $A=I$ (i.e., when $L$ is the Laplacian).

Going further, by invoking Theorem 4.1 we obtain that for each $\ell \in \mathbb{N}_{0}$ the $\ell$-th order Dirichlet boundary value problem (4.1) is well-posed when $L=\operatorname{div} A \nabla$. Moreover, the solution $u$ satisfies (4.3), and is given at each point $\left(x^{\prime}, t\right) \in \mathbb{R}_{+}^{n}$ by the formula

$$
u\left(x^{\prime}, t\right)=\frac{2 t}{\omega_{n-1} \sqrt{\operatorname{det}\left(A_{\mathrm{sym}}\right)}} \int_{\mathbb{R}^{n-1}} \frac{f\left(y^{\prime}\right)}{\left\langle\left(A_{\mathrm{sym}}\right)^{-1}\left(x^{\prime}-y^{\prime}, t\right),\left(x^{\prime}-y^{\prime}, t\right)\right\rangle^{\frac{n}{2}}} d y^{\prime} .
$$

5.2. The case of the Lamé system of elasticity. Recall that the Lamé operator in $\mathbb{R}^{n}$ has the form

$$
L u:=\mu \Delta u+(\lambda+\mu) \nabla \operatorname{div} u, \quad u=\left(u_{1}, \ldots, u_{n}\right) \in \mathscr{C}^{2},
$$

where the constants $\lambda, \mu \in \mathbb{R}$ (typically called Lamé moduli), are assumed to satisfy

$$
\mu>0 \text { and } 2 \mu+\lambda>0 .
$$

Condition (5.5) is equivalent to the demand that the Lamé system (5.4) satisfies the Legendre-Hadamard ellipticity condition (1.2). To illustrate the manner in which the Lamé system (5.4) may be written in infinitely many ways as in (1.1), for each $\theta \in \mathbb{R}$ introduce

$$
a_{r s}^{\alpha \beta}(\theta):=\mu \delta_{r s} \delta_{\alpha \beta}+(\lambda+\mu-\theta) \delta_{r \alpha} \delta_{s \beta}+\theta \delta_{r \beta} \delta_{s \alpha}, \quad 1 \leq \alpha, \beta, r, s \leq n .
$$

Then for each $\theta \in \mathbb{R}$ one can show that the Lamé operator (5.4) may be regarded as having the form (1.1) for the coefficient tensor $A=A(\theta):=\left(a_{r s}^{\alpha \beta}(\theta)\right)_{\substack{1 \leq r, s \leq n \\ 1 \leq \alpha, \beta \leq n}}$ with entries as in (5.6). In short, $A(\theta) \in \mathfrak{A}_{L}$ for each $\theta \in \mathbb{R}$.

Regarding the existence of a value for the parameter $\theta \in \mathbb{R}$ which makes $A(\theta)$ a distinguished coefficient tensor for the Lamé system, we note the following result.

Lemma 5.1. [7],[8] One has $A(\theta) \in \mathfrak{A}_{L}^{\text {dis }}$ if and only if $\theta=\frac{\mu(\lambda+\mu)}{3 \mu+\lambda}$. Moreover, corresponding to this value of $\theta$, the entries in $A(\theta)$ become for $\alpha, \beta, r, s \in\{1, \ldots, n\}$

$$
a_{r s}^{\alpha \beta}=\mu \delta_{r s} \delta_{\alpha \beta}+\frac{(\lambda+\mu)(2 \mu+\lambda)}{3 \mu+\lambda} \delta_{r \alpha} \delta_{s \beta}+\frac{\mu(\lambda+\mu)}{3 \mu+\lambda} \delta_{r \beta} \delta_{s \alpha} .
$$

In turn, for the choice of coefficient tensor as in (5.7), a straightforward calculation using the expression of the fundamental solution that can be found in e.g. [9] proves that (3.9) is satisfied if we consider, for every $\alpha, \beta \in\{1, \ldots, n\}$,

$$
k_{\alpha \beta}(x):=\frac{2 \mu}{3 \mu+\lambda} \frac{\delta_{\alpha \beta}}{\omega_{n-1}} \frac{1}{|x|^{n}}+\frac{\mu+\lambda}{3 \mu+\lambda} \frac{n}{\omega_{n-1}} \frac{x_{\alpha} x_{\beta}}{|x|^{n+2}}, \quad x \in \mathbb{R}^{n} \backslash\{0\} .
$$


Based on this and (3.16), we obtain that the unique Poisson kernel for the Lamé system (5.4) is the matrix-valued function $P=\left(P_{\alpha \beta}\right)_{1 \leq \alpha, \beta \leq n}: \mathbb{R}^{n-1} \rightarrow \mathbb{R}^{n \times n}$ whose entries are given for each $\alpha, \beta \in\{1, \ldots, n\}$ and $x^{\prime} \in \mathbb{R}^{n-1}$ by

$$
P_{\alpha \beta}\left(x^{\prime}\right)=\frac{4 \mu}{3 \mu+\lambda} \frac{\delta_{\alpha \beta}}{\omega_{n-1}} \frac{1}{\left(\left|x^{\prime}\right|^{2}+1\right)^{\frac{n}{2}}}+\frac{\mu+\lambda}{3 \mu+\lambda} \frac{2 n}{\omega_{n-1}} \frac{\left(x^{\prime}, 1\right)_{\alpha}\left(x^{\prime}, 1\right)_{\beta}}{\left(\left|x^{\prime}\right|^{2}+1\right)^{\frac{n+2}{2}}},
$$

In concert with Theorem 4.1, this analysis allows us to formulate the following well-posedness result for the $\ell$-th order Dirichlet problem for the Lamé system in the upper-half space.

Theorem 5.2. Assume that the Lamé moduli $\lambda, \mu$ satisfy (5.5). Then for every $p \in$ $(1, \infty)$, and for each $\ell \in \mathbb{N}_{0}$, the $\ell$-th order Dirichlet boundary value problem (4.1) is well-posed for the Lamé system (5.4). In addition, the solution $u=\left(u_{\alpha}\right)_{1 \leq \alpha \leq n}$ corresponding to the boundary datum $f=\left(f_{\beta}\right)_{1 \leq \beta \leq n} \in L_{\ell}^{p}\left(\mathbb{R}^{n-1}\right)$ is given by

$$
\begin{aligned}
u_{\alpha}\left(x^{\prime}, t\right)= & \frac{4 \mu}{3 \mu+\lambda} \frac{1}{\omega_{n-1}} \int_{\mathbb{R}^{n-1}} \frac{t}{\left(\left|x^{\prime}-y^{\prime}\right|^{2}+t^{2}\right)^{\frac{n}{2}}} f_{\alpha}\left(y^{\prime}\right) d y^{\prime} \\
& +\frac{\mu+\lambda}{3 \mu+\lambda} \frac{2 n}{\omega_{n-1}} \int_{\mathbb{R}^{n-1}} \frac{t\left(x^{\prime}-y^{\prime}, t\right)_{\alpha}\left(x^{\prime}-y^{\prime}, t\right)_{\beta}}{\left(\left|x^{\prime}-y^{\prime}\right|^{2}+t^{2}\right)^{\frac{n+2}{2}}} f_{\beta}\left(y^{\prime}\right) d y^{\prime},
\end{aligned}
$$

at each point $\left(x^{\prime}, t\right) \in \mathbb{R}_{+}^{n}$, and satisfies (4.3).

\section{REFERENCES}

[1] S. Agmon, A. Douglis, and L. Nirenberg, Estimates near the boundary for solutions of elliptic partial differential equations satisfying general boundary conditions, I, Comm. Pure Appl. Math., 12 (1959), 623-727.

[2] S. Agmon, A. Douglis, and L. Nirenberg, Estimates near the boundary for solutions of elliptic partial differential equations satisfying general boundary conditions, II, Comm. Pure Appl. Math., 17 (1964), 35-92.

[3] J. Garcia-Cuerva and J. Rubio de Francia, Weighted Norm Inequalities and Related Topics, North Holland, Amsterdam, 1985.

[4] L. Hörmander, The Analysis of Linear Partial Differential Operators. I. Distribution Theory and Fourier Analysis, Reprint of the second (1990) edition, Classics in Mathematics, Springer-Verlag, Berlin, 2003.

[5] F. John, Plane Waves and Spherical Means Applied to Partial Differential Equations, Interscience Publishers, New York-London, 1955.

[6] V.A. Kozlov, V.G. Maz'ya and J.Rossmann, Spectral Problems Associated with Corner Singularities of Solutions to Elliptic Equations, AMS, 2001.

[7] J.M. Martell, D. Mitrea, and M. Mitrea, Higher Order Regularity for Elliptic Boundary Value Problems, preprint, (2012).

[8] J.M. Martell, D. Mitrea, I. Mitrea, and M. Mitrea, Poisson kernels and boundary problems for elliptic systems in the upper-half space, preprint, (2012).

[9] D. Mitrea, Distributions, Partial Differential Equations, and Harmonic Analysis, Springer, Universitext, 2013.

[10] I. Mitrea and M. Mitrea, Multi-Layer Potentials and Boundary Problems for Higher-Order Elliptic Systems in Lipschitz Domains, Lecture Notes in Mathematics, Vol. 2063, Springer, 2013.

[11] C. B. Morrey, Second order elliptic systems of differential equations. Contributions to the theory of partial differential equations, Ann. Math. Studies, 33 (1954), 101-159.

[12] Z. Shapiro, On elliptical systems of partial differential equations, C. R. (Doklady) Acad. Sci. URSS (N.S.), 46 (1945). 
[13] V.A.Solonnikov, Estimates for solutions of general boundary value problems for elliptic systems, Doklady Akad. Nauk. SSSR, 151 (1963), 783-785 (Russian). English translation in Soviet Math., 4 (1963), 1089-1091.

[14] V.A. Solonnikov, General boundary value problems for systems elliptic in the sense of A. Douglis and L. Nirenberg. I, (Russian) Izv. Akad. Nauk SSSR, Ser. Mat., 28 (1964), 665-706.

[15] V.A. Solonnikov, General boundary value problems for systems elliptic in the sense of A. Douglis and L. Nirenberg. II, (Rusian) Trudy Mat. Inst. Steklov, Vol. 92 (1966), 233-297.

[16] E.M.Stein, Singular Integrals and Differentiability Properties of Functions, Princeton Mathematical Series, No. 30, Princeton University Press, Princeton, NJ, 1970.

[17] E.M.Stein, Harmonic Analysis: Real-Variable Methods, Orthogonality, and Oscillatory Integrals, Princeton Mathematical Series, Vol. 43, Monographs in Harmonic Analysis, III, Princeton University Press, Princeton, NJ, 1993.

José María Martell, Instituto de Ciencias Matemáticas CSIC-UAM-UC3M-UCM, ConSejo Superior de Investigaciones Científicas, C/ Nicolás Cabrera, 13-15, E-28049 Madrid, SPAIN

E-mail address: chema.martell@icmat.es

Dorina Mitrea, Department of Mathematics, University of Missouri, Columbia, MO 65211, USA

E-mail address: mitread@missouri.edu

Irina Mitrea, Department of Mathematics, Temple University, 1805 N. Broad Street, Philadelphia, PA 19122, USA

E-mail address: imitrea@temple.edu

Marius Mitrea, Department of Mathematics, University of Missouri, Columbia, MO 65211, USA

E-mail address: mitream@missouri.edu 